\title{
On periodicity and low complexity of infinite permutations
}

\author{
Dmitri G. Fon-Der-Flaass ${ }^{1 \dagger}$ and Anna E. Frid ${ }^{1 \ddagger}$ \\ ${ }^{1}$ Sobolev Institute of Mathematics SB RAS, Koptyug av. 4, 630090 Novosibirsk Russia
}

We define an infinite permutation as a sequence of reals taken up to the order, or, equivalently, as a linear ordering of a finite or countable set. Then we introduce and characterize periodic permutations; surprisingly, for each period $t$ there is an infinite number of distinct $t$-periodic permutations. At last, we introduce a complexity notion for permutations analogous to subword complexity for words, and consider the problem of minimal complexity of non-periodic permutations. Its answer is different for the right infinite and the bi-infinite case.

Keywords: infinite permutation, ordering, periodicity, complexity, subword complexity, Sturmian words

\section{Infinite permutations}

In this paper, we consider infinite permutations: a new object, naturally arising both from sequences of finite permutations and from sequences of reals. Furthermore, in many aspects infinite permutations are analogous to infinite words, and below we investigate similarity and distinction between these objects.

Let $S$ be a finite or countable ordered set: we shall consider $S$ equal either to $\mathbb{N}$, or to $\mathbb{Z}$, or to $\{1,2, \ldots, n\}$ for some integer $n$. Let $\mathcal{A}_{S}$ be the set of all sequences of pairwise distinct reals defined on $S$. Let us define an equivalence relation $\sim$ on $\mathcal{A}_{S}$ as follows: let $a, b \in \mathcal{A}_{S}$, where $a=\left\{a_{s}\right\}_{s \in S}$ and $b=\left\{b_{s}\right\}_{s \in S}$; then $a \sim b$ if and only if for all $s, r \in S$ the inequalities $a_{s}<a_{r}$ and $b_{s}<b_{r}$ hold or do not hold simultaneously. An equivalence class from $\mathcal{A}_{S} / \sim$ is called an $(S$-)permutation. Thus, an $S$-permutation $\alpha$ can be interpreted as a sequence of reals taken up to their order and defined by any its representative sequence $a$; in this case, we write $\alpha=\bar{a}$. In particular, a $\{1, \ldots, n\}$-permutation always has a representative with all values in $\{1, \ldots, n\}$, i. e., can be identified with a usual permutation from $S_{n}$.

In equivalent terms, a permutation can be defined as a linear ordering of $S$ which may differ from the "natural" one. To distinguish the two orders, we shall write $i<j, i, j \in S$, for the natural order, and $\alpha_{i}<\alpha_{j}$ for the order induced by the permutation.

A permutation can be represented also by a diagram where height of a point shows its position with respect to all other points.

\footnotetext{
${ }^{\dagger}$ Supported in part by RFBR grant 03-01-00796.

${ }^{\ddagger}$ Supported in part by Russian Science Support Foundation and RFBR grant 05-01-00364. 


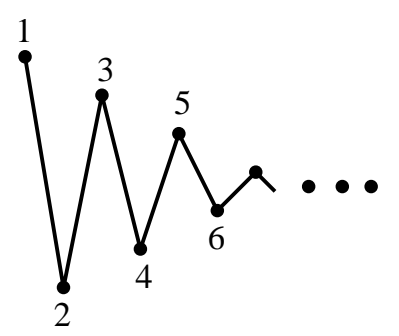

Fig. 1: A 2-periodic permutation

Example 1 Let $a=\left\{a_{i}\right\}_{i=1}^{\infty}=1,0,3 / 4,1 / 4,5 / 8,3 / 8, \ldots$ be a sequence from $\mathcal{A}_{\mathbb{N}}$ defined by

$$
a_{i}=\left\{\begin{array}{l}
1 / 2+1 / 2^{k}, i=2 k-1, \\
1 / 2-1 / 2^{k}, i=2 k
\end{array}\right.
$$

Then the permutation $\bar{a}=\alpha_{1}, \ldots, \alpha_{n}, \ldots$ can be defined also by the linear ordering $\alpha_{2}<\alpha_{4}<\cdots<$ $\alpha_{2 i}<\alpha_{2 i+2} \cdots<\alpha_{2 i+3}<\alpha_{2 i+1}<\cdots \alpha_{1}$ or by a diagram depicted at Fig. 1 .

Infinite permutations have been considered e. g. in [2]. In fact, any sequence of reals occurring in any problem can be considered as a representative of some infinite permutation. We can also define an infinite permutation as a limit of a sequence of finite (usual) permutations. In this paper, we study the properties of infinite permutations which are defined similarly to properties of infinite words, such as periodicity and "subword" complexity. As we shall see below, some of these properties look similarly to those of infinite words and some are not.

\section{Periodicity}

Let us say that a permutation $\alpha=\left\{\alpha_{s}\right\}_{s \in S}$ is $t$-periodic if for all $i$ and $j$ such that $i, j, i+t, j+t \in S$ the inequalities $\alpha_{i}<\alpha_{j}$ and $\alpha_{i+t}<\alpha_{j+t}$ are equivalent. An $\mathbb{N}$-permutation is called ultimately t-periodic if these inequalities are equivalent provided that $i, j>N_{0}$. Note that the permutation from Example 1 is 2-periodic.

Recall that a bi-infinite word $w=\cdots w_{-1} w_{0} w_{1} \cdots$ on a finite alphabet $\Sigma$ (with $w_{i} \in \Sigma$ ) is called $t$-periodic if $w_{i}=w_{i+t}$ for all $i$. Clearly, the number of $t$-periodic words is finite (and equal to $(\# \Sigma)^{t}$, if we fix the indices of symbols). So, it would be natural to conjecture that the number of $t$-periodic $\mathbb{Z}$-permutations is also not too large.

Surprisingly, for all $t \geq 2$ there exist infinitely many $t$-periodic $\mathbb{Z}$-permutations. A simple series of examples demonstrating it given by a series of representatives $\{a(n)\}_{n=1}^{\infty}$, where the sequence $a(n)$ is

$$
a(n)=\ldots-1,2 n-2,1,2 n, 3,2 n+2, \ldots
$$

Indeed, each of $a(n)$ is 2-periodic, and the first odd number which is greater than a given even one lies at the distance $2 n-1$ from it. So, all permutations $\overline{a(n)}$ are different.

Let us characterize all $t$-periodic $\mathbb{Z}$-permutations and give a way to code each of them.

Let $\alpha$ be a $t$-periodic $\mathbb{Z}$-permutation, and $a$ be any its representative sequence of reals. For $i=$ $0, \ldots, t-1$, consider the arithmetic progressions $A_{i}=\{x \in \mathbb{Z} \mid x \equiv i \bmod t\}$. Each of them is monotonic 
(increasing or decreasing) in the permutation. Let $S_{i}=\left(\inf a_{n}, \sup a_{n}\right)$ be the span of $A_{i}$, an open real interval. If two intervals $S_{i}$ and $S_{j}$ do not intersect, then the ordering between any element of $A_{i}$ and any element of $A_{j}$ is uniquely determined by the relative position of the intervals. Suppose that $S_{i} \cap S_{j} \neq \emptyset$. For definiteness, assume that the sequence $A_{i}$ is increasing (the other case is treated similarly). Since $S_{i}=\bigcup_{n \in \mathbb{Z}}\left[a_{i+n t}, a_{i+(n+1) t}\right]$, for some $m, n$ we have that $a_{i+n t}<a_{j+m t}<a_{i+(n+1) t}$. Then, by periodicity, for every $k \in \mathbb{Z}$ we have $a_{i+n t+k t}<a_{j+m t+k t}<a_{i+(n+1) t+k t}$. Therefore, $S_{i}=S_{j}$, both sequences are increasing, and between any two consecutive elements of one of them lies exactly one element of the other.

Now we can give a complete description of all $t$-periodic permutations. First, partition the set $I=$ $\{0, \ldots, t-1\}$ into one or more groups $I_{1}, \ldots, I_{k}$ (corresponding to disjoint intervals $S_{i}$ ). Define $\alpha_{x}<\alpha_{y}$ whenever $x \in A_{i}, y \in A_{j}, i \in I_{r}, j \in I_{s}$, and $r<s$. It remains only to define the ordering on elements of progressions belonging to the same group; for every group this can be done separately.

Take any group $J=I_{r}$. Choose whether it will be increasing or decreasing; that is, which will be every sequence $A_{i}$ from this group. Suppose that $J$ is increasing (the other case is similar). Take an arbitrary remainder $i \in J$. For every $j \neq i, j \in J$, there must be exactly one integer $n_{j}$ such that $\alpha_{i}<\alpha_{j+t n_{j}}<\alpha_{i+t}$. We can arbitrarily choose the values $n_{j}$, and then arbitrarily define the ordering of the numbers $a_{j+t n_{j}}$ for $j \neq i$. These data determine the permutation $\alpha$ between all elements of progressions belonging to the group.

The above arguments show that in this way we obtain every $t$-periodic permutation of $\mathbb{Z}$.

It remains to mention that each $t$-periodic $\mathbb{N}$-permutation uniquely determines a $t$-periodic $\mathbb{Z}$-permutation: for $x, y \in \mathbb{Z}$, choose $n$ such that $x+t n>0$ and $y+t n>0$, and set $\alpha_{x}<\alpha_{y}$ if and only if $\alpha_{x+t n}<\alpha_{y+t n}$. It is easy to check that the construction is correct, so, the characterization of periodic $\mathbb{N}$-permutations is induces by that for $\mathbb{Z}$-permutations.

At last, a natural question is: when does a periodic permutation admit a representation with all values in (a) $\mathbb{N}$ (b) $\mathbb{Z}$ ? We see that for a $\mathbb{N}$-permutation, (a) is equivalent to the situation when there is only one group $I=I_{1}$, and it is increasing; and (b) is possible if there is at most two groups $I_{1}$ and $I_{2}$, where $I_{1}$ is decreasing, $I_{2}$ is increasing, and each of them can be empty. For a $\mathbb{Z}$-permutation, (a) is impossible and (b) means that there is only one group $I=I_{1}$ which can be either decreasing or increasing.

\section{Factors and Complexity}

Let us say that a finite permutation $\alpha^{\prime}$ is a factor of an $S$-permutation $\alpha=\bar{a}$ if $\alpha^{\prime}=\overline{a_{k}, a_{k+1}, \ldots, a_{k+n-1}}$ for some $k \in S, n \geq 0$. This notion is analogous to that of factor, or subword, of a word $w=\cdots w_{1} w_{2} \cdots$, $w_{i} \in \Sigma$, which is defined as a word $w_{k} w_{k+1} \cdots w_{k+n-1}$ for some allowable $k$ and some $n \geq 0$.

The subword complexity $f_{w}(n)$ of a word $w$ is the number of its distinct factors of length $n$. This function gives a classical non-algorithmic way to define complexity of a sequence of symbols and is much explored (see survey [3]). In particular, clearly, it fits $1 \leq f_{w}(n) \leq(\# \Sigma)^{n}$ and is non-decreasing; the complexity of an ultimately periodic word is ultimately constant; we also have the following classical

Lemma 1 If an infinite word $w$ is not ultimately periodic, then $f_{w}(n)$ is strictly growing and fits $f_{w}(n) \geq$ $n+1$.

One-sided infinite words of minimal complexity are called Sturmian words [1] and have many non-trivial properties. The most famous of them is the Fibonacci word abaababaabaababaababaabaab $\cdots$ which can be constructed by iterating the morphism $a \mapsto a b, b \mapsto a$. Unlike a one-sided word, a bi-infinite word can have complexity $n+1$ even if it is quite simple, for example, equal to $\cdots 000010000 \cdots$. 
Analogously to the subword complexity of words, let us define complexity $f_{\alpha}(n)$ of a permutation $\alpha=$ $\bar{a}$ as the number of its factors of length $n$, that is, finite permutations of the form $\overline{a_{k}, a_{k+1}, \ldots, a_{k+n-1}}$. Clearly, this function fits $1 \leq f_{\alpha}(n) \leq n$ ! and is non-decreasing; however, unlike subword complexity for words, it is not obliged to be strictly growing even if the permutation is not periodic. In what follows we investigate what the "minimal" possible complexity is. The answer will differ for $\mathbb{N}$ - and $\mathbb{Z}$-permutations. However, the first lemma is unified and analogous to the classical (and nearly obvious) result for words.

Lemma 2 Let $\alpha$ be a $\mathbb{Z}\left(\mathbb{N}\right.$-)permutation; then $f_{\alpha}(n) \leq C$ if and only if $\alpha$ is periodic (ultimately periodic).

The difference with the situation for words appear when we try to find minimal possible complexity of a non-periodic permutation.

Theorem 1 For each unbounded growing function $g(n)$ there exists a not ultimately periodic $\mathbb{N}$-permutation $\alpha$ with $f_{\alpha}(n) \leq g(n)$ for all $n \geq n_{0}$.

SKETCH OF THE PROOF. The needed permutation can be defined by the inequalities $\alpha_{2 n-1}<\alpha_{2 n+1}$ and $\alpha_{2 n}<\alpha_{2 n+2}$ for all $n \geq 1$, and $\alpha_{2 n_{k}-2}<\alpha_{2 k-1}<\alpha_{2 n_{k}}$ for some sequence $\left\{n_{k}\right\}_{k=1}^{\infty}$ which grows sufficiently fast.

Theorem 2 For each non-periodic $\mathbb{Z}$-permutation $\alpha$ we have $f_{\alpha}(n) \geq n-C$ for some constant $C$ which can be arbitrarily large.

SKETCH OF THE PROOF. Let us consider a non-periodic $\mathbb{Z}$-permutation $\alpha$ such that $f_{\alpha}(n)<n$ for some $n$. Then there exists some $t \leq n$ such that $f_{\alpha}(t)=f_{\alpha}(t+1)=t$, and thus $\alpha$ is $t$-quasi-periodic, which means that $\left(\alpha_{i}<\alpha_{j}\right) \Leftrightarrow\left(\alpha_{i+t}<\alpha_{j+t}\right)$ for all $i, j$ such that $|j-i| \leq t$. In particular this means that each of the $t$ arithmetic progressions of difference $t$, i. e., permutations $A_{i}=\ldots, \alpha_{i-t}, \alpha_{i}, \alpha_{i+t}, \ldots$, $i=1, \ldots, t$, is monotonic. Let us say that the permutations $A_{i}$ and $A_{j}, i<j$, are adjusted if the permutation $A_{i j}=\ldots, \alpha_{i-t}, \alpha_{j-t}, \alpha_{i}, \alpha_{j}, \alpha_{i+t}, \alpha_{j+t}, \ldots$ is periodic. It can be seen that $\alpha$ is periodic if and only if all pairs of $A_{i}$ are adjusted. So, the fact that $\alpha$ is not periodic means that so is some $\beta=A_{i j}$.

First, suppose that $A_{i}$ is increasing and $A_{j}$ is decreasing (or vise versa). If the intervals in which their representations lie do not intersect, then $\beta$ is 2-periodic, a contradiction. If they intersect, then there exist some $k$ and $l$ such that either $\alpha_{j+(k+1) t}<\alpha_{i+l t}<\alpha_{j+k t}$, or $\alpha_{i+k t}<\alpha_{j+l t}<\alpha_{i+(k+1) t}$. In both cases, all factors of $\alpha$ of length at least $t$ which include all the three mentioned elements are distinct. Since they are $n-c$, where $c$ is the maximal distance between two of three indices, we have $f_{\alpha}(n) \geq n-c$, which is what we needed.

Now suppose that both $A_{i}$ and $A_{j}$ are increasing (the case when they are decreasing is analogous). Suppose that $\alpha_{i}<\alpha_{j}$ (and thus $\alpha_{i+k t}<\alpha_{j+k t}$ for all $k$ due to quasi-periodicity). Let us consider a series of bi-infinite words $w(n), n=0,1, \ldots$, defined by

$$
w(n)_{k}=\left\{\begin{array}{l}
a, \text { if } \alpha_{i+(k+n) t}<\alpha_{j+k t}, \\
b, \text { otherwise. }
\end{array}\right.
$$

By the definition, $w(0)=\cdots$ aaaaa $\cdots$. If all $w(n)$ are also equal to $\cdots$ aaaaa $\cdots$, then $\beta$ is 2-periodic, a contradiction. So, the letter $b$ occurs in some $w(n)$.

Suppose that some $w(m)$ is non-periodic. Then its subword complexity is at least $n+1$ due to Lemma 1. Counting the number of factors of $\alpha$ which include $\alpha_{j+k t}$ and $\alpha_{i+(k+m+n) t}$ for some $k$ (i. e., the information about a factor of $w(m)$ of length $n+1)$, we once again obtain $f_{\alpha}(n) \geq n-c$, where $c$ 


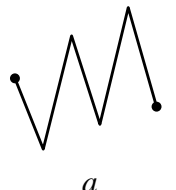

$a$

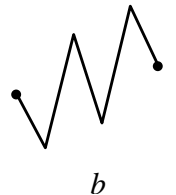

$b$

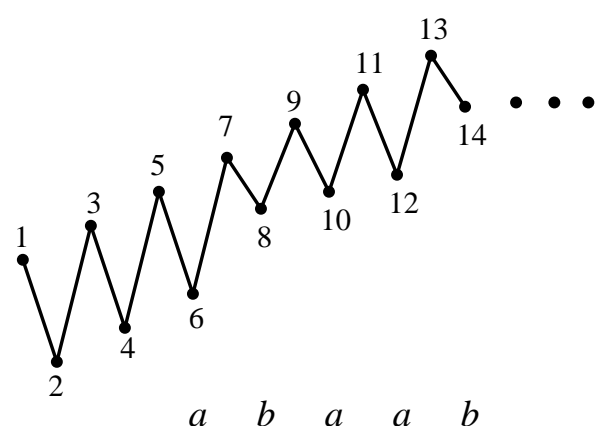

Fig. 2: A $\mathbb{Z}$-permutation of complexity at least $n-3$ based on the Fibonacci word

depends on $m$. An example of such a permutation $\alpha$ of complexity $f_{\alpha}(n) \geq n-3$, which is built on the base of $w(3)$ equal to the (bi-infinite analogue of) the Fibonacci word is depicted at Fig. 2. Note that we have some freedom in the relations between, e. g., $\alpha_{5}$ and $\alpha_{12}$, so it is easy to make the complexity greater than $n-3$.

It remains to consider the case when all $w(n)$ are periodic and some of them (say, $w(m)$ ) contains the letter $b$. Let $w(m)$ be $p$-periodic; so, letters $b$ occur in it at most at the distance $p$ from each other. Note also that $w(k)_{i}=b$ implies $w(k+1)_{i-1}=w(k+1)_{i}=b$, and thus $w(n)=\cdots b b b b b \cdots$ for all $n \geq m+p$. So, there is just a finite number of sequences $w(n)$ not equal to $\cdots b b b b b \cdots$; all of them are periodic. We see that $\beta$ is periodic with the period equal to the $\mathrm{lcm}$ of all periods of $w(n)$. A contradiction.

\section{Acknowledgements}

We are deeply grateful to Sergej Avgustinovich who attracted our attention to infinite permutations and stated some of the problems solved above.

\section{References}

[1] J. Berstel, P. Séébold, Sturmian words, in: M. Lothaire, Algebraic Combinatorics on Words, Cambridge University Press, 2002. P. 40-97.

[2] J. A. Davis, R. C. Entringer, R. L. Graham, and G. J. Simmons, On permutations containing no long arithmetic progressions, Acta Arithmetica 34 (1977), 81-90.

[3] S. Ferenczi, Complexity of sequences and dynamical systems, Discrete Math. 206 (1999), 145-154. 
